\title{
Particle Mass Limits in Minimal and Nonminimal Supersymmetric Models 1
}

\author{
P. N. Pandita \\ Department of Physics, North Eastern Hill University \\ Umshing-Mawkynroh, Shillong 793 022, India
}

\begin{abstract}
A review of the Higgs and neutralino sector of supersymmetric models is presented. This includes the upper limit on the mass of the lightest Higgs boson in the Minimal Supersymmetric Standard Model, as well as models based on the Standard Model gauge group $S U(2)_{L} \times U(1)_{Y}$ with extended Higgs sectors. We then discuss the Higgs sector of left-right supersymmetric models, which conserve R-parity as a consequence of gauge invariance, and present a calculable upper bound on the mass of the lightest Higgs boson in these models. We also discuss the neutralino sector of general supersymmetric models based on the SM gauge group. We show that, as a consequence of gauge coupling unification, an upper bound on the mass of the lightest neutralino as a function of the gluino mass can be obtained.
\end{abstract}

\footnotetext{
${ }^{1}$ Invited talk given at the Fifth Workshop on High Energy Physics Phenomenology, Inter-University Centre for Astronomy and Astrophysics, Pune, India, January 12 - 26, 1998
} 


\section{Introduction}

The Standard Model (SM) of elementary particle physics, which is extremely successful in describing the experimental data, is based on two fundamental principles, i.e. gauge invariance and Higgs mechanism. From recent experiments it is clear that strong and electroweak interactions are described by an $S U(3)_{C} \times S U(2)_{L} \times U(1)_{Y}$ gauge theory. On the other hand, little is known about the mechanism of electroweak gauge symmetry breaking. In the Standard Model, the electroweak $S U(2)_{L} \times U(1)_{Y}$ gauge symmetry is broken through the Higgs mechanism via the vacuum expectation value (VEV) of the neutral component of the Higgs doublet [1], leaving behind a remanant in the form of an elementary scalar particle, the Higgs boson, which has so far not been observed. Apart from the fact that the VEV is an arbitrary parameter in the SM, the mass parameter of the Higgs field suffers from quadratic divergences, making the weak scale unstable under radiative corrections [1].

One of the central problems of particle physics is, then, to understand how the electroweak scale associated with the mass of the $\mathrm{W}$ boson is generated, and why is it so small as compared to the Planck scale associated with the Newton's constant. Supersymmetry [2, 3, 4] is at present the only known framework in which the weak scale is stable under radiative corrections, although it does not explain how such a small scale arises in the first place. As such, considerable importance attaches to the study of supersymmetric models, especially the Minimal Supersymmetric Standard Model (MSSM), based on the gauge group $S U(3)_{C} \times S U(2)_{L} \times U(1)_{Y}$, with two Higgs doublet superfields $\left(H_{1}, H_{2}\right)$ with

opposite hypercharges: $Y\left(H_{1}\right)=-1, Y\left(H_{2}\right)=+1$, so as to generate masses for up- and down-type quarks (and leptons), and to cancel gauge anomalies. In general, supersymmetric extensions of SM have extended Higgs sectors leading to a rich penomenology of Higgs bosons. 
In this talk I will discuss the Higgs sector of supersymmetric models. This will include the Minimal Supersymmetric Standard Model, as well as models having extended Higgs sectors, based on the SM gauge group. I will then explore supersymmetric models based on extended gauge groups, e.g. the left-right gauge group $S U(2)_{L} \times S U(2)_{R} \times U(1)_{B-L}$, pointing out the relevance of extended gauge symmetries in the context of supersymmetric models, and discuss the Higgs sector of these models.

In supersymmetric gauge theories, each fermion and boson of the Standard Model is accompanied by its supersymmetric partner, transforming in an identical manner under the gauge group. In supersymmetric theories with R-parity conservation, the lightest supersymmetric particle (LSP) is expected to be the lightest neutralino, which is the lightest mixture of the fermionic partners of the neutral Higgs and neutral electroweak gauge bosons. The lightest neutralino, being the LSP, is the end product of any process involving supersymmetric particles in the final state. In this talk, I will also discuss the neutralino sector of the general supersymmetric models based on the SM gauge group.

\section{The Higgs Sector of the Minimal Supersymmetric Standard Model}

The Higgs sector of the Minimal Supersymmetric Standard Model, based on the gauge group $S U(3)_{C} \times S U(2)_{L} \times U(1)_{Y}$, contains two Higgs doublet superfields $\left(H_{1}, H_{2}\right)$ with opposite hypercharges: $Y\left(H_{1}\right)=-1, Y\left(H_{2}\right)=+1$, so as to generate masses for up- and down-type quarks (and leptons), and to cancel gauge anomalies. The tree level scalar potential of Higgs bosons in MSSM can be wriitten as

$$
\begin{aligned}
V_{H} & =m_{1}^{2}\left|H_{1}\right|^{2}+m_{2}^{2}\left|H_{2}\right|^{2}-m_{3}^{2}\left(H_{1} \cdot H_{2}+\text { h.c. }\right) \\
& +\frac{g^{2}+g^{\prime 2}}{8}\left(\left|H_{1}\right|^{2}-\left|H_{2}\right|^{2}\right)^{2}+\frac{1}{2} g^{2}\left|H_{1}^{*} H_{2}\right|^{2},
\end{aligned}
$$


where $g$ and $g^{\prime}$ are the $S U(2)_{L}$ and $U(1)_{Y}$ gauge couplings, respectively. We note that, as a consequence of gauge invariance and supersymmetry, the quartic couplings of Higgs bosons in MSSM are fixed in terms of electroweak gauge couplings. After spontaneous symmetry breaking induced by the neutral components of $H_{1}$ and $H_{2}$ obtaining vacuum expectation values, $\left\langle H_{1}\right\rangle=v_{1},\left\langle H_{2}\right\rangle=v_{2}, \tan \beta=v_{2} / v_{1}$, the MSSM contains two neutral $C P$-even $\left(h^{0}, H^{0}\right)$, one neutral $C P$-odd $(A)$, and two charged $\left(H^{ \pm}\right)$Higgs bosons [1]. Although gauge invariance and supersymmetry fix the quartic couplings of the Higgs bosons in MSSM in terms of $S U(2)_{L}$ and $U(1)_{Y}$ gauge couplings, there still remain two independent parameters which describe the Higgs sector of the MSSM. These are usually chosen to be $\tan \beta$ and $m_{A}$, the mass of the $C P$-odd Higgs boson. All the Higgs masses and the Higgs couplings in MSSM can be described (at tree level) in terms of these two parameters. From (2.1) it follows that the lightest CP-even neutral Higgs boson has a tree level upper bound of $M_{Z}$ (the mass of $Z^{0}$ boson) on its mass [5, 6]. However, radiative corrections [7, 8, 9, 10] weaken this tree level upper bound. In the one-loop effective potential approximation, the radiatively corrected squared mass matrix for the $C P$-even Higgs bosons can be written as [1]

$$
\begin{aligned}
\mathcal{M}^{2}= & {\left[\begin{array}{cc}
m_{A}^{2} \sin ^{2} \beta+m_{Z}^{2} \cos ^{2} \beta & -\left(m_{Z}^{2}+m_{A}^{2}\right) \sin \beta \cos \beta \\
-\left(m_{Z}^{2}+m_{A}^{2}\right) \sin \beta \cos \beta & m_{A}^{2} \cos ^{2} \beta+m_{Z}^{2} \sin ^{2} \beta
\end{array}\right] } \\
& +\frac{3 g^{2}}{16 \pi^{2} m_{W}^{2}}\left[\begin{array}{cc}
\Delta_{11} & \Delta_{12} \\
\Delta_{12} & \Delta_{22}
\end{array}\right]
\end{aligned}
$$

where the second matrix represents the radiative corrections.

The functions $\Delta_{i j}$ depend on, besides the top- and bottom-quark masses, the Higgs bilinear parameter $\mu$ in the super-potential, the soft supersymmetry breaking trilinear couplings $\left(A_{t}, A_{b}\right)$ and soft scalar masses $\left(m_{Q}, m_{U}, m_{D}\right)$, as well as $\tan \beta$. We shall ignore the b-quark mass effects in $\Delta_{i j}$ in the following, which is a reasonable approximation for 
moderate values of $\tan \beta \leq 20-30$. Furthermore, we shall assume, as is often done,

$$
\begin{aligned}
A & \equiv A_{t}=A_{b}, \\
\tilde{m} & \equiv m_{Q}=m_{U}=m_{D} .
\end{aligned}
$$

With these approximations we can write $\left(m_{t}\right.$ is the top quark mass) [1]

$$
\begin{aligned}
\Delta_{11} & =\frac{m_{t}^{4}}{\sin ^{2} \beta}\left(\frac{\mu(A+\mu \cot \beta)}{m_{\tilde{t}_{1}}^{2}-m_{\tilde{t}_{2}}^{2}}\right)^{2} g\left(m_{\tilde{t}_{1}}^{2}, m_{\tilde{t}_{2}}^{2}\right), \\
\Delta_{22} & =\frac{m_{t}^{4}}{\sin ^{2} \beta}\left(\log \frac{m_{\tilde{t}_{1}}^{2} m_{\tilde{t}_{2}}^{2}}{m_{t}^{4}}+\frac{2 A(A+\mu \cot \beta)}{m_{\tilde{t}_{1}}^{2}-m_{\tilde{t}_{2}}^{2}} \log \frac{m_{\tilde{t}_{1}}^{2}}{m_{\tilde{t}_{2}}^{2}}\right) \\
& +\frac{m_{t}^{4}}{\sin ^{2} \beta}\left(\frac{\mu(A+\mu \cot \beta)}{m_{\tilde{t}_{1}}^{2}-m_{\tilde{t}_{2}}^{2}}\right)^{2} g\left(m_{\tilde{t}_{1}}^{2}, m_{\tilde{t}_{2}}^{2}\right), \\
\Delta_{12} & =\frac{m_{t}^{4}}{\sin ^{2} \beta} \frac{\mu(A+\mu \cot \beta)}{m_{\tilde{t}_{1}}^{2}-m_{\tilde{t}_{2}}^{2}}\left(\log \frac{m_{\tilde{t}_{1}}^{2}}{m_{\tilde{t}_{2}}^{2}}+\frac{A(A+\mu \cot \beta)}{m_{\tilde{t}_{1}}^{2}-m_{\tilde{t}_{2}}^{2}} g\left(m_{\tilde{t}_{1}}^{2}, m_{\tilde{t}_{2}}^{2}\right)\right),
\end{aligned}
$$

where $m_{\tilde{t}_{1}}^{2}$ and $m_{\tilde{t}_{2}}^{2}$ are squared stop masses, and $g\left(m_{\tilde{t}_{1}}^{2}, m_{\tilde{t}_{2}}^{2}\right)$ is a function of stop masses, given by (we have ignored the small $D$-term contributions to the stop masses)

$$
\begin{aligned}
m_{\tilde{t}_{1,2}}^{2} & =m_{t}^{2}+\tilde{m}^{2} \pm m_{t}(A+\mu \cot \beta), \\
g\left(m_{\tilde{t}_{1}}^{2}, m_{\tilde{t}_{2}}^{2}\right) & =2-\frac{m_{\tilde{t}_{1}}^{2}+m_{\tilde{t}_{2}}^{2}}{m_{\tilde{t}_{1}}^{2}-m_{\tilde{t}_{2}}^{2}} \log \frac{m_{\tilde{t}_{1}}^{2}}{m_{\tilde{t}_{2}}^{2}}
\end{aligned}
$$

The one-loop radiatively corrected masses $\left(m_{h}, m_{H} ; m_{h}<m_{H}\right)$ of the $C P$-even Higgs bosons $\left(h^{0}, H^{0}\right)$ can be obtained by diagonalizing the $2 \times 2$ mass matrix (2.2). The radiative corrections are, in general, positive, and they shift the mass of the lightest Higgs boson upwards from its tree-level value. We show in Fig.(1) the resulting masses of the CP-even Higgs bosons, $m_{h}$ and $m_{H}$, as well as the charged Higgs boson mass, as a function of $m_{A}$ for two different values of $\tan \beta=1.5,30$. With a wider range of parameter values, or when the squark mass scale is taken to be smaller, the dependence on $\mu$ and $\tan \beta$ can be more dramatic [12, 13, 14. 
Although radiative corrections can be appreciable, these depend only logarithmically on the SUSY breaking scale, and are, therefore, under control. In particular, the mass of the lightest neutral scalar is bounded from above:

$$
m_{h}^{2} \leq M_{Z}^{2} \cos ^{2}(2 \beta)+\epsilon\left(m_{t}, m_{\tilde{t}_{1}}, m_{\tilde{t}_{2}}, A_{t}, \mu, \cdots\right),
$$

where $\epsilon$ parameterizes the effect of the radiative corrections described above. Note that $\epsilon$ is approximately independent of $\tan \beta$; for large $m_{A}, m_{t}=175 \mathrm{GeV}$ and $m_{\tilde{t}_{1,2}}=1 \mathrm{TeV}$ it

amounts to about $0.9 M_{W}^{2}\left(1.6 M_{W}^{2}\right)$ for no (maximal) stop mixing. It is important to note that the bound (2.9) can only be saturated for large $m_{A}$. This rsults in an upper bound of about $125-135 \mathrm{GeV}$ on the one-loop radiatively corrected mass of the lightest Higgs boson of MSSM [15].

The Higgs mass falls rapidly at small values of $\tan \beta$. Since the LEP experiments are obtaining lower bounds on the mass of the lightest Higgs boson, they are beginning to rule out significant parts of the small-tan $\beta$ parameter space, depending on the model assumptions. For $\tan \beta>1$, ALEPH finds $m_{h}>62.5 \mathrm{GeV}$ at $95 \%$ C.L. [16]. [For a recent discussion on how the lower allowed value of $\tan \beta$ depends on some of the model parameters, see Ref. [17].]

The two-loop corrections to the lightest higgs mass are typically $\mathcal{O}(20 \%)$ of the oneloop corrections, and are negative. For the dominant two-loop radiative corrections to the Higgs sector of MSSM, see, e.g. [18, 19].

\section{The Higgs Sector of the Non-Minimal Supersym- metric Standard Model}

If we concentrate on the Higgs sector, the MSSM is special because the Higgs self-couplings at the tree level are completely determined by gauge couplings. Although the MSSM is 
the simplest, and, thus, the most widely, studied model, there are several viable extensions of the supersymmetric version of the SM. A simplest extension of the Higgs sector of the MSSM is to postulate the existence of a $S U(2)_{L} \times U(1)_{Y}$ Higgs singlet superfield $N$ in the spectrum [20]. This model, referred to as the Non-Minimal Supersymmetric Standard Model (NMSSM), does not destroy the unification of coupling constants achieved in the MSSM, since the new light particles do not carry SM quantum numbers.

Even if we restrict ourselves to purely cubic terms in the superpotential $W$, gauge symmetry allows one to introduce two different Higgs self-couplings:

$$
W_{\text {Higgs }}=\lambda N H_{1} H_{2}-\frac{k}{3} N^{3}
$$

where we have used the notation of Ref. [21]. Together with the corresponding soft breaking terms, there are six free parameters in the Higgs sector, even after we fix the sum of the squares of the VEV's of the $S U(2)$ doublets to reproduce the known mass of the $Z$ boson. Moreover, the spectrum now contains three neutral $\mathrm{CP}$-even fields $H_{i}$ and two $\mathrm{CP}$-odd fields $A_{i}$ in addition to the charged Higgs field $H^{ \pm}$.

Because of the presence of the trilinear coupling proportional $\lambda$ in the superpotential (3.10), the tree-level Higgs-boson self-coupling in the NMSSM depends on $\lambda$ as well as the gauge couplings. Nevertheless, one can still derive [22, 23, 24, 25, 26, 27, 28, an upper bound on the mass of the lightest CP-even Higgs boson of the NMSSM. Including radiative corrections, one has

$$
m_{H_{1}}^{2} \leq M_{Z}^{2} \cos ^{2}(2 \beta)+\frac{2 \lambda^{2} M_{W}^{2}}{g^{2}} \sin ^{2}(2 \beta)+\epsilon,
$$

where $\epsilon$ parametrizes the effect of radiative corrections, which are similar in nature to the corresponding corrections in the MSSM. Because of the presence of the term proportional to the coupling $\lambda$ in (3.11), no definite upper bound on the mass of the lightest CP-even Higgs boson in NMSSM can be given unless a further assumption on the strength of this 
coupling is made. If we require all dimensionless coupling constants to remain perturbative upto the GUT scale, we can calculate the the upper bound on the lightest CP-even Higgsboson mass. The resulting upper bound is shown in Fig.(2), and is compared with the corresponding bound in the MSSM [25, 26, 29, 30]. The top-quark-mass dependence of the upper bound is not significant compared to the MSSM case because the maximally allowed value of $\lambda$ is larger(smaller) for a smaller (larger) top mass.

One can study the implications of introducing higher dimensional Higgs representations on the upper bound for the mass of the lightest Higgs boson in supersymmetric models. Because of the presence of the additional trilinear Yukawa couplings, a tight constraint on the mass of the lightest Hiss boson need not a priori hold in such extensions of MSSM based on the gauge group $S U(2)_{L} \times U(1)_{Y}$ with an extended Higgs sector. Nevertheless, it has been shown that the upper bound on the lightest Higgs boson mass in these models depends only on the weak scale and dimensionless coupling constants (and only logarithmically on the SUSY breaking scale), and is calculable if all the couplings remain perturbative below some scale $\Lambda$ [31, 32]. This upper bound can vary between $150 \mathrm{GeV}$ to $165 \mathrm{GeV}$ depending on the Higgs structure of the underlying supersymmetric model. Thus, nonobservation of a light Higgs boson below this upper bound will rule out an entire class of supersymmetric models based on the gauge group $S U(2)_{L} \times U(1)_{Y}$.

\section{Supersymmetric Models with Extended gauge Groups}

The existence of upper bound on the lightest Higgs boson mass in MSSM (with arbitrary Higgs sectors) has been investigated in a situation where the underlying supersymmetric model respects baryon $(B)$ and lepton $(L)$ number conservation. However, it is well known that gauge invariance, supersymmetry and renormalizibility allow $B$ and $L$ violating terms in the superpotentioal of the MSSM [33, 34]. The strength of these lepton and baryon 
number violating terms is, however, severely limited by phenomenological [35, 36, 37, 38, 39, 40, 41, 42, 43] and cosmological [44, 45] constraints. Indeed, unless the strength of baryon-number-violating term is less than $10^{-13}$, it will lead to contradiction with the present lower limits on the lifetime of the proton. The usual strategy to prevent the appearance of $B$ and $L$ violating couplings in MSSM is to invoke a discrete $Z_{2}$ symmetry 46] known as matter parity, or R-parity. The matter parity of each superfield may be defined as

$$
\text { (matter parity) } \equiv(-1)^{3(B-L)} \text {. }
$$

The multiplicative conservation of matter parity forbids all the renormalizable $B$ and $L$ violating terms in the superpotential of MSSM. Equivalently, the R-parity of any component field is defined by $R_{p}=(-1)^{3(B-L)+2 S}$, where $S$ is the spin of the field. Since $(-1)^{2 S}$ is conserved in any Lorentz-invariant interaction, matter parity conservation and R-parity conservation are equivalent. Conservation of R-parity then immediately implies that superpartners can be produced only in pairs, and that the lightest supersymmetric particle (LSP) is absolutely stable.

Although the Minimal Supersymmetric Standard Model with R-parity conservation can provide a description of nature which is consistent with all known observations, the assumption of $R_{p}$ conservation appears to be ad hoc, since it is not required for the internal consistency of MSSM. Furthermore, all global symmetries, discrete or continuous, could be violated by the Planck scale physics effects [47, 48, 49, 50, 51]. The problem becomes acute for low energy supersymmetric models, because $B$ and $L$ are no longer automatic symmetries of the Lagrangian, as they are in the Standard Model. It is, therefore, more appealing to have an supersymmetric theory where R-parity is related to a gauge symmetry, and its conservation is automatic because of the invariance of the underlying theory under this gauge symmetry. Fortunately, there is a compelling 
scenario which does automatically provide for exact R-parity conservation due to a deeper principle. Indeed, $R_{p}$ conservation follows automatically in certain theories with gauged $(B-L)$, as is suggested by the fact that matter parity is simply a $Z_{2}$ subgroup of ( $B-$ $L)$. It has been noted by several authors [52, 53, 54] that if the gauge symmetry of MSSM is extended to $S U(2)_{L} \times U(1)_{I_{3 R}} \times U(1)_{B-L}$, or $S U(2)_{L} \times S U(2)_{R} \times U(1)_{B-L}$, the theory becomes automatically R-parity conserving. Such a left-right supersymmetric theory (SLRM) solves the problems of explicit $B$ and $L$ violation of MSSM, and has received much attention recently [55, 56, 57, 58, 59, 60, 61, 62, 63. . Of course left-right theories are also interesting in their own right, for among other appealing features, they offer a simple and natural explanation for the smallness of neutrino mass through the so called see-saw mechanism 64, 65].

Such a naturally R-parity conserving theory necessarily involves the extension of the Standard Model gauge group, and since the extended gauge symmetry has to be broken, it involves a "new scale", the scale of left-right symmetry breaking, beyond the SUSY and $S U(2)_{L} \times U(1)_{Y}$ breaking scales of MSSM. It is, therefore, important to ask whether the upper bound on the lightest Higgs mass in naturally R-parity conserving theories depends on the scale of the breakdown of the extended gauge group. We now consider the question of the mass of the lightest Higgs boson in left-right supersymmetric models so as to answer this question [66].

We begin by recalling some basic features of the left-right supersymmetric models used in our study. Further details can be found, e.g., in [62, 66]. The quark and lepton doublets are included in $Q(2,1,1 / 3) ; Q^{c}(1,2,-1 / 3) ; L(2,1,-1) ; L^{c}(1,2,1)$, where $Q$ and $Q^{c}$ denote the left- and right-handed quark superfields and similarly for the leptons $L$ and $L^{c}$. Note that since left- and right-handed fermions are placed symmetrically in doublets, also the right-handed neutrinos are included. The Higgs superfields consist of 
$\Delta_{L}(3,1,-2) ; \Delta_{R}(1,3,-2) ; \delta_{L}(3,1,2) ; \delta_{R}(1,3,2) ; \Phi(2,2,0) ; \chi(2,2,0)$. The numbers in the parentheses denote the representation content of the fields under the gauge group $S U(2)_{L} \times S U(2)_{R} \times U(1)_{B-L}$. The two $S U(2)_{R}$ Higgs triplet superfields $\Delta_{R}(1,3,-2)$ and $\delta_{R}(1,3,2)$ with opposite $(B-L)$ are necessary to break the left-right symmetry spontaneously, and to cancel triangle gauge anomalies due to the fermionic superpartners of Higgs bosons. The left-right model also contains the $S U(2)_{L}$ triplets $\Delta_{L}$ and $\delta_{L}$ in order to make the Lagrangian fully symmetric under the $L \leftrightarrow R$ transformation, although these are not needed phenomenologically for the symmetry breaking or the see-saw mechanism [64, 65] for neutrino mass generation. The two bi-doublet Higgs superfields $\Phi$ and $\chi$ are required in order to break the $S U(2)_{L} \times U(1)_{Y}$ and to generate a non-trivial Kobayashi-Maskawa matrix.

The most general gauge invariant superpotential for the model can be written as

$$
\begin{aligned}
W= & h_{\phi Q} Q^{T} i \tau_{2} \Phi Q^{c}+h_{\chi Q} Q^{T} i \tau_{2} \chi Q^{c}+h_{\phi L} L^{T} i \tau_{2} \Phi L^{c}+h_{\chi L} L^{T} i \tau_{2} \chi L^{c} \\
& +h_{\delta_{L}} L^{T} i \tau_{2} \delta_{L} L+h_{\Delta_{R}} L^{c T} i \tau_{2} \Delta_{R} L^{c}+\mu_{1} \operatorname{Tr}\left(i \tau_{2} \Phi^{T} i \tau_{2} \chi\right)+\mu_{1}^{\prime} \operatorname{Tr}\left(i \tau_{2} \Phi^{T} i \tau_{2} \Phi\right) \\
& +\mu_{1}^{\prime \prime} \operatorname{Tr}\left(i \tau_{2} \chi^{T} i \tau_{2} \chi\right)+\operatorname{Tr}\left(\mu_{2 L} \Delta_{L} \delta_{L}+\mu_{2 R} \Delta_{R} \delta_{R}\right) .
\end{aligned}
$$

From the superpotential, the scalar potential, and the CP-even Higgs mass matrix, can be calculated via a standard procedure. Using the fact that for any Hermetian matrix its smallest eigenvalue must be smaller than that of its upper left corner $2 \times 2$ submatrix, we obtain from this mass matrix the upper bound on the mass of the lightest Higgs boson in the left-right supersymmetric model:

$$
m_{h}^{2} \leq \frac{1}{2}\left(g_{L}^{2}+g_{R}^{2}\right)\left(\kappa_{1}^{2}+\kappa_{2}^{2}\right) \cos ^{2} 2 \beta
$$

where $g_{L}$ and $g_{R}$ are the $S U(2)_{L}$ and $S U(2)_{R}$ gauge couplings, respectively, and $\kappa_{1}$ and $\kappa_{2}$ are the VEV's of the neutral components of $\Phi(2,2,0)$ and $\chi(2,2,0)$, respectively, with $\tan \beta=\kappa_{2} / \kappa_{1}$. We note that the upper bound (4.14) is not only independent of the 
supersymmetry breaking parameters (as in the case of supersymmetric models based on $\left.S U(2)_{L} \times U(1)_{Y}\right)$, but it is independent of the $S U(2)_{R}$ breaking scale, which, a priori, can be very large, and also independent of any R-parity breaking vacuum expectation value. The upper bound is controlled by $\left(\kappa_{1}^{2}+\kappa_{2}^{2}\right)$ and the dimensionless gauge couplings $\left(g_{L}\right.$ and $\left.g_{R}\right)$ only. Since the former is essentially fixed by the electroweak scale, the gauge couplings $g_{L}$ and $g_{R}$ determine the bound (4.14). Since the right-handed gauge coupling $g_{R}$ is not known, the upper bound on the right-hand side of (4.14) comes from the requirement [66] that the left-right supersymmetric model remains perturbative below some scale $\Lambda$. The resulting tree level upper bound is shown in Fig.(3). The tree-level bound can be considerably larger than in MSSM, if the difference between the high scale $\Lambda$ and the intermediate scale $M_{R}$ is small. The radiative corrections to the upper bound from top-stop and bottom-sbottom sector are sizable and of the same form as in the MSSM. In Fig.(4), we show the radiatively corrected upper bound as a function of top quark mass in the range $150<m_{t}<200 \mathrm{GeV}$, and compare it with the corresponding upper bound in the MSSM. The upper bound increases with increasing $M_{R}$ scale, and becomes less restrictive as this scale is increased. For $M_{R}=10 \mathrm{TeV}$ and $m_{\text {top }}=175$ $\mathrm{GeV}$, the bound remains below $155 \mathrm{GeV}$ while for $M_{R}=10^{10} \mathrm{GeV}$ it remains below 175 $\mathrm{GeV}$. It is seen that the mass limits, except for large $\mu_{1}, \mu_{1}^{\prime}, \mu_{1}^{\prime \prime}$, are somewhat higher in SUSYLR than in the MSSM.

\section{The Neutralino Sector of Supersymmetric Models}

In supersymmetric theories with R-parity conservation, the lightest neutralino is expected to be the lightest supersymmetric particle. In MSSM the fermionic partners of the Higgs bosons mix with the fermionic partners of the gauge bosons to produce four neutralino states $\tilde{\chi}_{i}^{0}, i=1,2,3,4$, and two chargino states $\tilde{\chi}_{i}^{ \pm}, i=1,2$. An upper bound on the 
squared mass of the lightest neutralino $\chi_{1}^{0}$ can be obtained by using the fact that the smallest eigenvalue of the mass squared matrix of the neutralinos is smaller than the smallest eigenvalue of its upper left 2 × 2 submatrix

$$
\left[\begin{array}{cc}
M_{1}^{2}+M_{Z}^{2} \sin ^{2} \theta_{W} & -M_{Z}^{2} \sin \theta_{W} \cos \theta_{W} \\
-M_{z}^{2} \sin \theta_{W} \cos \theta_{W} & M_{2}^{2}+M_{Z}^{2} \cos ^{2} \theta_{W}
\end{array}\right]
$$

thereby resulting in the upper bound 67]

$$
M_{\chi_{1}^{0}}^{2} \leq \min \left(M_{1}^{2}+M_{Z}^{2} \sin ^{2} \theta_{W}, M_{2}^{2}+M_{Z}^{2} \cos ^{2} \theta_{W}\right)
$$

We note that the uppwer bound (5.16) is controlled by, in addition to $M_{Z}$ and $\theta_{W}$, the soft SUSY breaking gaugino masses, $M_{1}$ and $M_{2}$. This is in contrast to the Higgs sector of MSSM, where the corresponding bound on the (tree level) mass of the lightest Higgs boson is controlled by $M_{Z}$, and not by supersymmetry breaking masses. However, the upper bound can become meaningful in theories with gauge coupling unification.

We recall that, as a consequence of the renormalization group equations (RGEs) satisfied by the gauge couplings and the gaugino masses in the MSSM, we have $\left(\alpha_{i}=\right.$ $\left.g_{i}^{2} / 4 \pi, \alpha_{U}=g_{U}^{2} / 4 \pi\right)$

$$
M_{1}\left(M_{Z}\right) / \alpha_{1}\left(M_{Z}\right)=M_{2}\left(M_{Z}\right) / \alpha_{2}\left(M_{Z}\right)=M_{3}\left(M_{Z}\right) / \alpha_{3}\left(M_{Z}\right)=m_{1 / 2} / \alpha_{U}
$$

where $M_{1 / 2}$ is the common gaugino mass at the grand unification scale, and $\alpha_{U}$ is the unified coupling constant. It is important to note that (15.17), which is a consequence of one-loop renormalization group equations, is valid in any grand unified theory irrespective of the particle content. It reduces the three gaugino mass parameters to one, which we choose to be the gluino mass $m_{\tilde{g}}$, which is equal to $\left|M_{3}\right|$. This results in an upper bound on the mass of the lightest neutralino as a function of the gluino mass:

$$
M_{\chi_{1}^{0}}^{2} \leq M_{1}^{2}+M_{Z}^{2} \sin ^{2} \theta_{W} \simeq\left(0.02 m_{\tilde{g}}^{2}+1924.5\right) \mathrm{GeV}^{2}
$$


For a gluino mass of $200 \mathrm{GeV}$, the upper bound (5.18) on the mass of the lightest neutralino is $52 \mathrm{GeV}$, which increases to $148 \mathrm{GeV}$ for a gluino mass of $1 \mathrm{TeV}$. The radiative corrections to the upper bound can vary between $5 \%$ and $20 \%$ depending upon the composition of the lightest neutralino.

Although the upper bound (5.18) on the lightest neutralino mass has been obtained in the MSSM, a similar upper bound can be obtained in the more general models based on the gauge group $S U(2)_{L} \times U(1)_{Y}$ with an extended Higgs sector 68. Numerically the upper bound in these extended models can be typically higher than the one in MSSM.

\section{Acknowledgements}

The author would like to thank the organizers of the Fifth Workshop on Particle Physics Phenomenology (WHEPP5) for inviting him to the Workshop, and for the hospitality. This work is supported by Department of Science and Technology under project No. $\mathrm{SP} / \mathrm{S} 2 / \mathrm{K}-17 / 94$.

\section{References}

[1] Gunion, J.F., Haber, H.E., Kane, G.L. and Dawson, S.: 1990, 'The Higgs Hunters Guide', Addison-Wesley, Reading, MA, U.S.A.

[2] Haber, H.E. and Kane, G.L.: 1985, Phys. Rept. Vol. no. 117, p. 75

[3] Nilles, H.P.: 1984, Phys. Rept. Vol. no. 110, p. 1

[4] Nath, P., Arnowitt, R. and Chamseddine, A.H.: 1984, 'Applied N=1 supergravity', World Scientific, Singapore

[5] Inoue, K. et al:: 1982, Prog. Theor. Phys. Vol. no. 67, p. 1889 
[6] Flores, R.A. and Sher, M.: 1983, Ann. Phys. Vol. no. 148, p. 95

[7] Okada, Y., Yamaguchi, M. and Yanagida, T.: 1991, Prog. Theor. Phys. Vol. no. 85, p. 1

[8] Ellis, J., Ridolfi, G. and Zwirner, F.: 1991, Phys. Lett. Vol. no. B257, p. 83

[9] Haber, H.E. and Hempfling, R.: 1991, Phys. Rev. Lett. Vol. no. 66, p. 1815

[10] Barbieri, R., Frigeni, M. and Caravaglios, F.: 1991, Phys. Lett. Vol. no. B258, p. 167

[11] Ellis, J., Ridolfi, G. and Zwirner, F.: 1991, Phys. Lett. Vol. no. B262, p. 477

[12] Kileng, B., Osland, P. and Pandita, P.N.: 1996, Z. Phys. Vol. no. C71, p. 87

[13] Kileng, B., Osland, P. and Pandita, P.N.: 1996, in Xth International Workshop: High Energy Physics and Quantum Field Theory, Proceedings of the conference, Zvenigorod, Russia, September 20-26, 1995, ed. B.B. Levtchenko and V.I. Savrin (Moscow University Press, Moscow, 1996), p. 167 [hep-ph/9601284]

[14] Kileng, B., Osland, P. and Pandita, P.N.: 1997, in Quantum Systems: New Trends and Methods, Proceedings of the International Workshop, Minsk, Belarus, June 3-7, 1996, eds. Y. S. Kim, L. M. Tomil'chik, I. D. Feranchuk and A. Z. Gazizov (World Scientific, Singapore, 1997), p. 231 [hep-ph/9608315]

[15] Rosiek, J. and Sopczak, A.: 1995, Phys. Lett. Vol. no. B341, p. 419

[16] Barate, R., et al. (ALEPH Collaboration): 1997, Phys. Lett. Vol. no. B412, p. 173

[17] Carena, M., Chankowski, P.H., Pokorski, S. and Wagner, C.E.M.: 1998, CERNTH/98-148, [hep-ph/9805349] 
[18] Carena, M., Espinosa, J.R., Quirós, M. and Wagner, C.E.M.: 1995, Phys. Lett. Vol. no. B355, p. 209

[19] Carena, M., Quirós, M. and Wagner, C.E.M.: 1996, Nucl. Phys. Vol. no. B461, p. 407

[20] Nilles, H.P., Srednicki, M. and Wyler D.: 1983, Phys. Lett. Vol. no. B120, p. 346

[21] Ellis, J., Gunion, J.F., Haber, H.E., Roszkowski, L. and Zwirner, F.: 1989, Phys. Rev. Vol. no. D39, p. 844

[22] Drees, M.: 1989, Int. J. Mod. Phys. Vol. no. A4, p. 3635

[23] Binetruy, P. and Savoy, C.A.: 1992, it Phys. Lett. Vol. no. B277, p. 453

[24] Moroi, T and Okada, Y.: 1992, Phys. Lett. Vol. no. B295, p. 73

[25] Pandita, P.N.: 1993, Phys. Lett. Vol. no. B318, p. 338

[26] Pandita, P.N.: 1993, Z. Phys. Vol. no. C59, p. 575

[27] Elliott, T., King, S.F. and White, P.L.: 1993, Phys. Lett. Vol. no. B305, p. 71

[28] Ellwanger, U.: 1993, Phys. Lett. Vol. no. B303, p. 271

[29] Ananthanarayan, B. and Pandita, P.N.: 1995, Phys. Lett. Vol. no. B353, p. 70

[30] Okada, Y.: 1996, Talk Presented at the Workshop on Physics and Experiments with Linear Colliders, Morioka-Appi, Japan, hep-ph/9602283

[31] Kane, G.L., Kolda, C. and Wells, J.D.: 1993, Phys. Rev. Lett. Vol. no. 70, p. 2686

[32] Espinosa, J.R. and Quiros, M.: 1993, Phys. Lett. Vol. no. B302, p. 51

[33] Weinberg, S.: 1982, Phys. Rev. Vol. no. D26, p. 287 
[34] Sakai, N. and Yanagida, T.: 1982 Nucl. Phys. Vol. no. B197, p. 533

[35] Zwirner, F.: 1984 Phys. Lett. Vol. no. B132, p. 103

[36] Hall, L.J. and Suzuki, M.: 1984, Nucl. Phys. Vol. no. B231, p. 419

[37] Lee, I.H.: 1984, Nucl. Phys. Vol. no. B246, p. 120

[38] Dawson, S.: 1985, Nucl. Phys. Vol. no. B261, p. 297

[39] Barbieri, R. and A. Masiero, A.: 1986, Nucl. Phys. Vol. no. B267, p. 679

[40] Dimopoulos, S. and Hall, L.J.: 1988, Phys. Lett. Vol. no. B207, p. 210

[41] Barger, V., Giudice, G.F. and Han, T.: 1989, Phys. Rev. Vol. no. D40, p. 2987

[42] Dreiner, H. and Ross, G.G.: 1991, Nucl. Phys. Vol. no. B365, p. 597

[43] Smirnov, A.Y. and Vissani, F.: 1996, Nucl. Phys. Vol. no. B460, p. 37

[44] Campbell, B.A., et al.: 1991, Phys. Lett. Vol. no. B256, p. 457

[45] Fischler, W., et al.: 1991, Phys. Lett. Vol. no. B258, p. 45

[46] Farrar, G.R. and Fayet, P.: 1978, Phys. Lett. Vol. no. B76, p. 575

[47] Giddings, S. and Strominger, A.: 1988, Nucl. Phys. Vol. no. B307, p. 854

[48] Coleman, S.: 1988, Nucl. Phys. Vol. no. B310, p. 643

[49] Preskill, J. and Krauss, L.M.: 1990, Nucl. Phys. Vol. no. B341, p. 50

[50] Holman, R., et al.: 1992, Phys. Lett. Vol. no. B282, p. 132

[51] Kamionkowski, M. and March-Russell, J.: 1992, Phys. Lett. Vol. no. B282, p. 137

[52] Mohapatra, R.N.: 1986, Phys. Rev. Vol. no. D34, p. 3457 
[53] Font, A., Ibanez, L.E. and Quevedo, F.: 1989, Phys. Lett. Vol. no. B228, p. 79

[54] Martin, S.P.: 1992, Phys. Rev. Vol. no. D46, p. 2769

[55] Cvetic, M. and Pati, J.C.: 1984, Phys. Lett. Vol. no. B135, p. 57

[56] Francis, R.M., Frank, M. and Kalman, C.S.: 1991, Phys. Rev. Vol. no. D43, p. 2369

[57] Francis, R.M., Kalman, C.S. and Saif, H.F.: 1991, Z. Phys. Vol. no. C59, p. 655

[58] Kuchimanchi, R. and Mohapatra, R.N.: 1993, Phys. Rev. Vol. no. D48, p. 4352

[59] Huitu, K., Maalampi, J. and Raidal, M.: 1994: Nucl. Phys. Vol. no. B420, p. 449

[60] Huitu, K., Maalampi, J. and Raidal, M.: 1994: Phys. Lett. Vol. no. B320, p. 60

[61] Huitu, K., Maalampi, J.: 1995, Phys. Lett. Vol. no. B344, p. 217

[62] Huitu, K., Pandita, P.N. and Puolamaki, K.: 1997, Phys. Lett. Vol. no. B415, p. 156

[63] Aulakh, C.S., Benakli, K. and Senjanovic, G.: 1997, Phys. Rev. Lett. Vol. no. 79, p. 2188 ,

[64] Gell-Mann, M., Ramond, P. and Slansky, R.: 1979, Supergravity, eds. P. van Niewenhuizen and D.Z. Freedman (North Holland, 1979)

[65] Yanagida, T.: 1979, Proceedings of Workshop on Unified Theory and Baryon Number in the Universe, eds. O.Sawada and A. Sugamoto (KEK 1979)

[66] Huitu, K., Pandita, P.N. and Puolamaki, K.: 1998, Phys. Lett. Vol. no. B423, p. 97 
[67] Pandita, P.N.: 1996, Phys. Rev. Vol. no. D53, p. 566

[68] Pandita, P.N.: 1997, Int. J. Mod. Phys., (to appear) [hep-ph/9701411] 


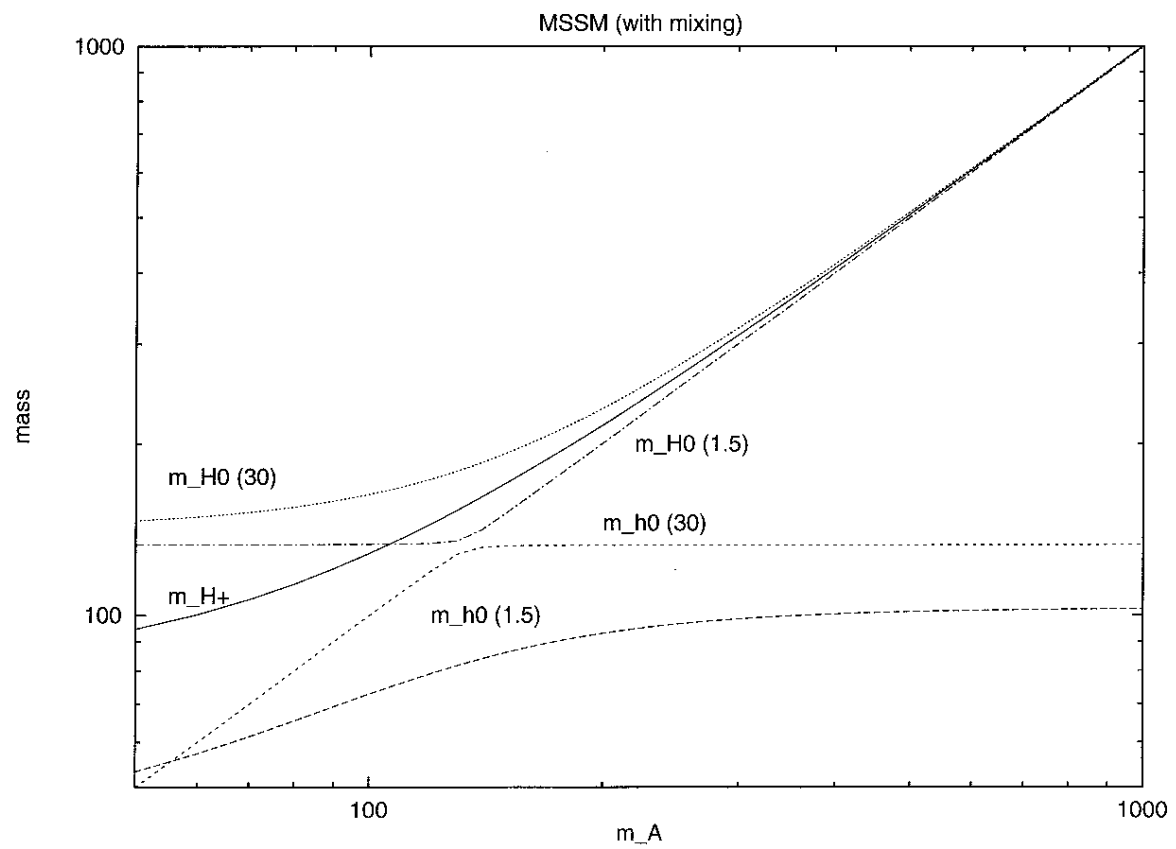

Figure 1: Masses of the CP-even Higgs bosons $h^{0}, H^{0}$ and of the charged Higgs particles $H^{ \pm}$as a function of the CP-odd Higgs mass $m_{A}$ for two values of $\tan \beta=1.5,30$. 


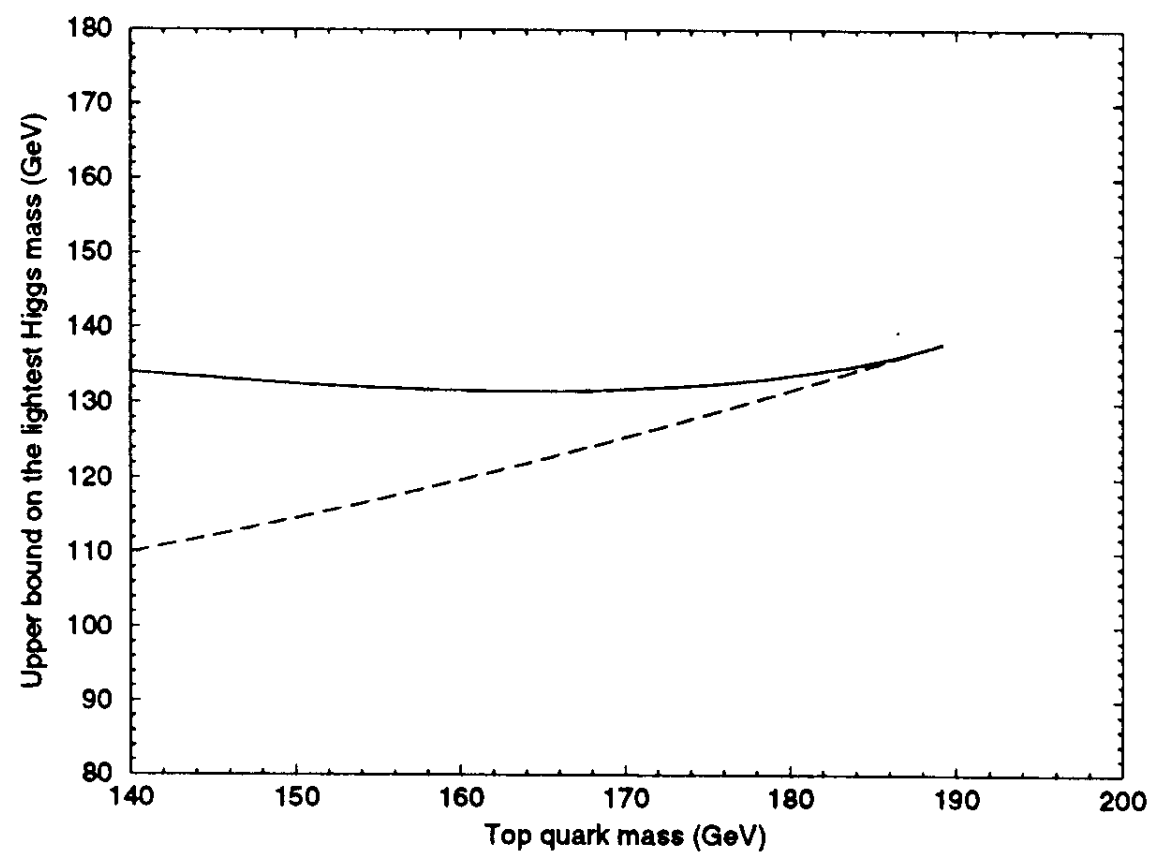

Figure 2: The upper bound on the mass of the lightest CP-even Higgs boson in the NonMinimal Supersymmetric Standard Model (solid line). We have taken stop mass to be 1 $\mathrm{TeV}$. The dotted line shows the corresponding upper bound in the MSSM. 


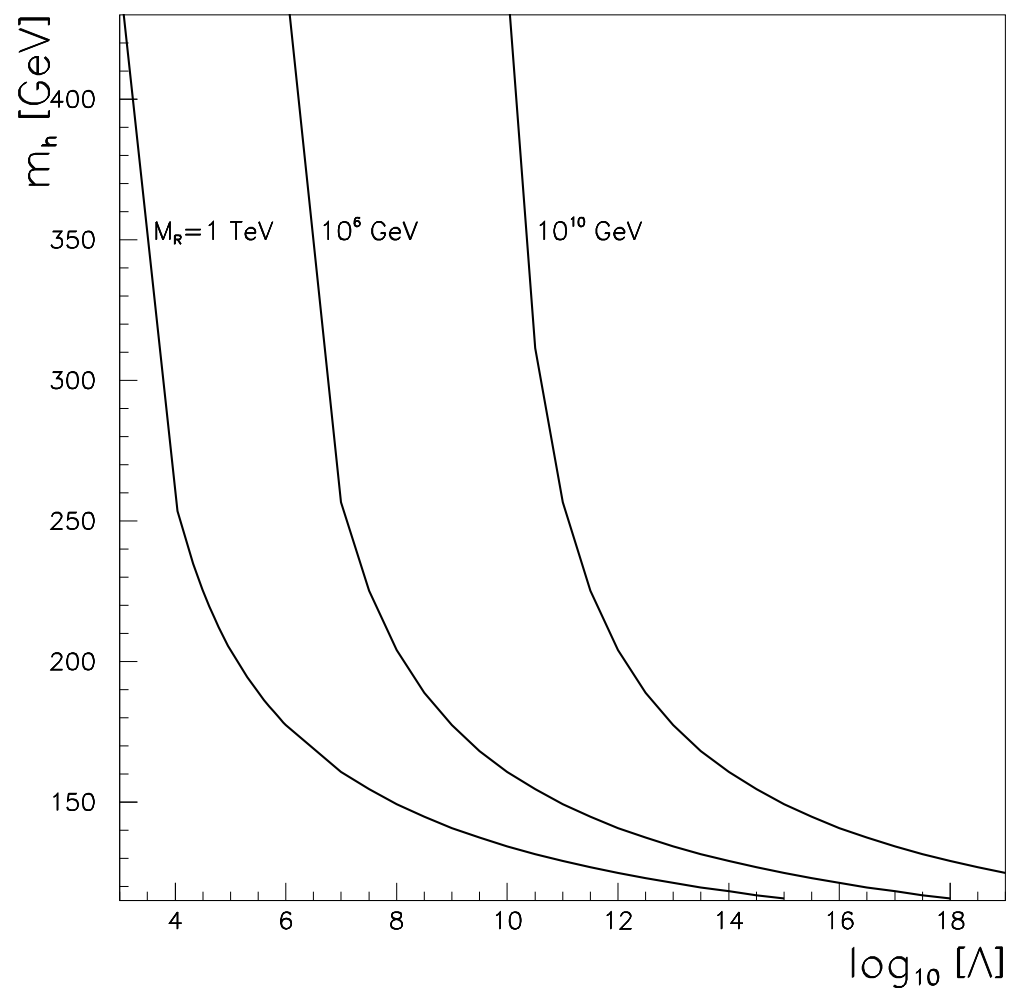

Figure 3: The tree-level upper bound on the lightest Higgs mass as a function of the scale $\Lambda$ up to which the $g_{R}$ coupling remains perturbative. The plotted $S U(2)_{R} \times U(1)_{B-L}$ breaking scales are $M_{R}=1 \mathrm{TeV}, 10^{6} \mathrm{GeV}$ and $10^{10} \mathrm{GeV}$. 

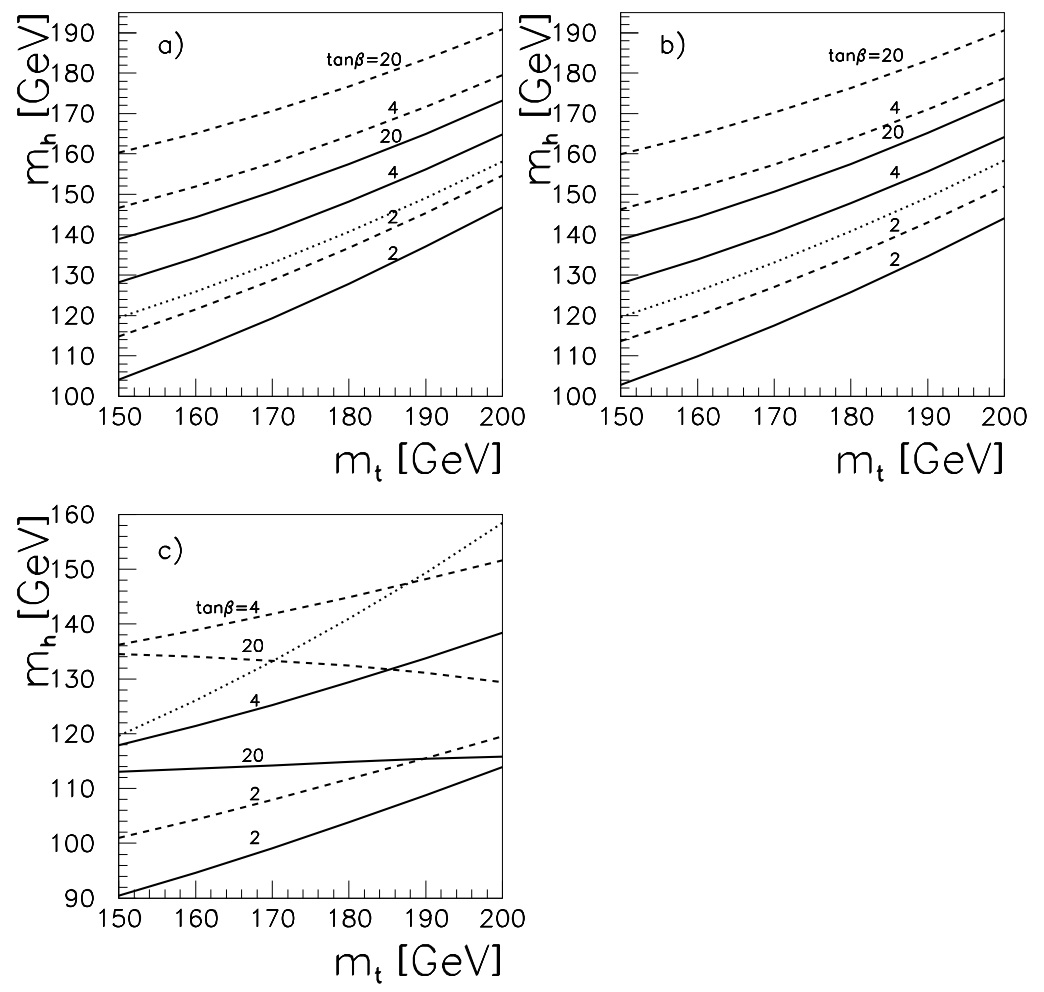

Figure 4: The radiatively corrected upper limit on the mass of the lightest Higgs boson as a function of $m_{t}$ with $\Lambda=10^{16} \mathrm{GeV}$ and $A_{t}=A_{b}=1=\mathrm{TeV}$. The solid line corresponds to the $S U(2)_{R}$ scale of $10 \mathrm{TeV}$ and the dashed line to the $S U(2)_{R}$ scale of $10^{10} \mathrm{GeV}$. The dotted curve corresponds to MSSM limit for $\tan \beta=20$ and $\mu=\mu_{1}$. In a) $\mu_{1}=\mu_{1}^{\prime}=$ $\mu_{1}^{\prime \prime}=0$, in b) $\mu_{1}=\mu_{1}^{\prime}=\mu_{1}^{\prime \prime}=500 \mathrm{GeV}$, and in c) $\mu_{1}=\mu_{1}^{\prime}=\mu_{1}^{\prime \prime}=1000 \mathrm{GeV}$. 